\title{
On a new extended half-discrete Hilbert's inequality involving partial sums
}

\author{
Xing Shou Huang ${ }^{1 *}$, Ricai Luo ${ }^{1}$ and Bicheng Yang ${ }^{2}$
}

"Correspondence: hxs803@126.com

'School of Mathematics and

Statistics, Hechi University, Yizhou,

P.R. China

Full list of author information is

available at the end of the article

\begin{abstract}
By applying the weight functions, the idea of introducing parameters, and Euler-Maclaurin summation formula, a new extended half-discrete Hilbert's inequality with the homogeneous kernel and the beta, gamma function is given. The equivalent statements of the best possible constant factor related to a few parameters are considered. As applications, a corollary about the case of the non-homogeneous kernel and some particular cases are obtained.
\end{abstract}

MSC: $26 \mathrm{D} 15$

Keywords: Weight function; Half-discrete Hilbert's inequality; Parameter; Euler-Maclaurin summation formula; Gamma function; Beta function

\section{Introduction}

If $0<\sum_{m=1}^{\infty} a_{m}^{2}<\infty$ and $0<\sum_{n=1}^{\infty} b_{n}^{2}<\infty$, then we have the following discrete Hilbert's inequality with the best possible constant factor $\pi$ (cf. [1], Theorem 315):

$$
\sum_{m=1}^{\infty} \sum_{n=1}^{\infty} \frac{a_{m} b_{n}}{m+n}<\pi\left(\sum_{m=1}^{\infty} a_{m}^{2} \sum_{n=1}^{\infty} b_{n}^{2}\right)^{1 / 2} .
$$

Assuming that $0<\int_{0}^{\infty} f^{2}(x) d x<\infty$ and $0<\int_{0}^{\infty} g^{2}(y) d y<\infty$, we still have the following Hilbert's integral inequality (cf. [1], Theorem 316):

$$
\int_{0}^{\infty} \int_{0}^{\infty} \frac{f(x) g(y)}{x+y} d x d y<\pi\left(\int_{0}^{\infty} f^{2}(x) d x \int_{0}^{\infty} g^{2}(y) d y\right)^{1 / 2}
$$

where the constant factor $\pi$ is the best possible. Inequalities (1) and (2) play an important role in the analysis and its applications (cf. [2-13]).

We still have the following half-discrete Hilbert-type inequality (cf. [1], Theorem 351): If $K(x)(x>0)$ is a decreasing function, $p>1, \frac{1}{p}+\frac{1}{q}=1,0<\phi(s)=\int_{0}^{\infty} K(x) x^{s-1} d x<\infty$, $f(x) \geq 0,0<\int_{0}^{\infty} f^{p}(x) d x<\infty$, then

$$
\sum_{n=1}^{\infty} n^{p-2}\left(\int_{0}^{\infty} K(n x) f(x) d x\right)^{p}<\phi^{p}\left(\frac{1}{q}\right) \int_{0}^{\infty} f^{p}(x) d x .
$$

In recent years, some new extensions of (3) have been provided by [14-19].

(c) The Author(s) 2020. This article is licensed under a Creative Commons Attribution 4.0 International License, which permits use, sharing, adaptation, distribution and reproduction in any medium or format, as long as you give appropriate credit to the original author(s) and the source, provide a link to the Creative Commons licence, and indicate if changes were made. The images or other third party material in this article are included in the article's Creative Commons licence, unless indicated otherwise in a credit line to the material. If material is not included in the article's Creative Commons licence and your intended use is not permitted by statutory regulation or exceeds the permitted use, you will need to obtain permission directly from the copyright holder. To view a copy of this licence, visit http://creativecommons.org/licenses/by/4.0/. 
In 2006, by using the Euler-Maclaurin summation formula, Krnic et al. [20] gave an extension of (1) with the kernel $\frac{1}{(m+n)^{\lambda}}(0<\lambda \leq 4)$; and in 2019, according to the results of [20], Adiyasuren et al. [21] considered an extension of (1) involving the partial sums.

In 2016-2017, by applying the weight functions, Hong [22, 23] considered some equivalent statements of the extensions of (1) and (2) with a few parameters. Some similar interested works were provided by [24-26].

In this paper, according to the way of $[21,22]$, by the use of the weight functions, the idea of introducing parameters and the Euler-Maclaurin summation formula, a new extended half-discrete Hilbert's inequality with the homogeneous kernel $\frac{1}{(x+n)^{\lambda}}(0<\lambda \leq 26)$ and the beta, gamma function is given. The equivalent statements of the best possible constant factor related to a few parameters are considered. As applications, a corollary about the case of non-homogeneous kernel and some particular cases are also obtained.

\section{Some lemmas}

In what follows, we assume that $p>1, \frac{1}{p}+\frac{1}{q}=1, \lambda \in(-2,26], \lambda_{2} \in(-1,1], \lambda_{1}, \lambda_{2} \in(-1, \lambda+$ $1), f(x) \geq 0, f \in L^{1}\left(R_{+}\right)\left(R_{+}=(0, \infty)\right), a_{n} \geq 0(n \in \mathbb{N}=\{1,2, \ldots\}),\left\{a_{n}\right\}_{n=1}^{\infty} \in l^{1}$,

$$
F(x):=\int_{0}^{x} f(t) d t \quad(x \geq 0), \quad A_{n}:=\sum_{k=1}^{n} a_{k} \quad(n \in \mathbf{N})
$$

such that

$$
0<\int_{0}^{\infty} x^{p\left[1-\left(\frac{\lambda+1-\lambda_{2}}{p}+\frac{\lambda_{1}+1}{q}\right)\right]-1} F^{p}(x) d x<\infty \quad \text { and } \quad 0<\sum_{n=1}^{\infty} n^{q\left[1-\left(\frac{\lambda_{2}+1}{p}+\frac{\lambda+1-\lambda_{1}}{q}\right)\right]-1} A_{n}^{q}<\infty
$$

By the definition of the gamma function, for $\lambda, x>0, n \in \mathbf{N}$, the following equality holds:

$$
\frac{1}{(x+n)^{\lambda}}=\frac{1}{\Gamma(\lambda)} \int_{0}^{\infty} t^{\lambda-1} e^{-(x+n) t} d t
$$

Lemma 1 For $t>0$, we have

$$
\begin{aligned}
& \sum_{n=1}^{\infty} e^{-t n} a_{n} \leq t \sum_{n=1}^{\infty} e^{-t n} A_{n} \\
& \int_{0}^{\infty} e^{-t x} f(x) d x=t \int_{0}^{\infty} e^{-t x} F(x) d x
\end{aligned}
$$

Proof Since $\left\{a_{n}\right\}_{n=1}^{\infty} \in l^{1}$, we find $\lim _{n \rightarrow \infty} A_{n}=\sum_{i=1}^{\infty} a_{i} \in[0, \infty)$. Using Abel's summation by parts formula and the inequality $1-e^{-t} \leq t$, we have (cf. [21])

$$
\begin{aligned}
\sum_{n=1}^{\infty} e^{-t n} a_{n} & =\lim _{n \rightarrow \infty} e^{-t(n+1)} A_{n}+\sum_{n=1}^{\infty}\left[e^{-t n}-e^{-t(n+1)}\right] A_{n} \\
& =\left(1-e^{-t}\right) \sum_{n=1}^{\infty} e^{-t n} A_{n} \leq t \sum_{n=1}^{\infty} e^{-t n} A_{n}
\end{aligned}
$$


namely, inequality (5) follows. For $f \in L^{1}\left(R_{+}\right), F(0)=0, F(\infty) \in[0, \infty)$, we find

$$
\begin{aligned}
\int_{0}^{\infty} e^{-t x} f(x) d x & =\int_{0}^{\infty} e^{-t x} d F(x)=\left.e^{-t x} F(x)\right|_{0} ^{\infty}-\int_{0}^{\infty} F(x) d e^{-t x} \\
& =t \int_{0}^{\infty} e^{-t x} F(x) d x
\end{aligned}
$$

and then expression (6) follows.

Lemma 2 For $1<s \leq 28, \sigma \in(0,2] \cap(0, s)$, define the following weight function:

$$
\varpi(\sigma, x):=x^{s-\sigma} \sum_{n=1}^{\infty} \frac{n^{\sigma-1}}{(x+n)^{s}} \quad\left(x \in \mathrm{R}_{+}\right)
$$

We have the following inequality:

$$
\varpi(\sigma, x)<B(\sigma, s-\sigma) \quad\left(x \in \mathrm{R}_{+}\right) .
$$

Proof We set function $g(t):=\frac{t^{\sigma-1}}{(x+t)^{s}}(t>0)$. Using the Euler-Maclaurin summation formula (cf. [20]), for $\rho(t):=t-[t]-\frac{1}{2}$, we have

$$
\begin{aligned}
& \sum_{n=1}^{\infty} g(n)=\int_{1}^{\infty} g(t) d t+\frac{1}{2} g(1)+\int_{1}^{\infty} \rho(t) g^{\prime}(t) d t=\int_{0}^{\infty} g(t) d t-h(\sigma, s), \\
& h(\sigma, s):=\int_{0}^{1} g(t) d t-\frac{1}{2} g(1)-\int_{1}^{\infty} \rho(t) g^{\prime}(t) d t .
\end{aligned}
$$

We obtain $-\frac{1}{2} g(1)=\frac{-1}{2(x+1)^{s}}$. Integrating by parts, it follows that

$$
\begin{aligned}
\int_{0}^{1} g(t) d t & =\int_{0}^{1} \frac{t^{\sigma-1}}{(x+t)^{s}} d t=\frac{1}{\sigma} \int_{0}^{1} \frac{d t^{\sigma}}{(x+t)^{s}}=\left.\frac{1}{\sigma} \frac{t^{\sigma}}{(x+t)^{s}}\right|_{0} ^{1}+\frac{s}{\sigma} \int_{0}^{1} \frac{t^{\sigma} d t}{(x+t)^{s+1}} \\
& =\frac{1}{\sigma} \frac{1}{(x+1)^{s}}+\frac{s}{\sigma(\sigma+1)} \int_{0}^{1} \frac{d t^{\sigma+1}}{(x+t)^{s+1}} \\
& >\frac{1}{\sigma} \frac{1}{(x+1)^{s}}+\frac{s}{\sigma(\sigma+1)}\left[\frac{t^{\sigma+1}}{(x+t)^{s+1}}\right]_{0}^{1}+\frac{s(s+1)}{\sigma(\sigma+1)} \int_{0}^{1} \frac{t^{\sigma+1}}{(x+1)^{s+2}} d t \\
& =\frac{1}{\sigma} \frac{1}{(x+1)^{s}}+\frac{s}{\sigma(\sigma+1)} \frac{1}{(x+1)^{s+1}}+\frac{s(s+1)}{\sigma(\sigma+1)(\sigma+2)} \frac{1}{(x+1)^{s+2}} .
\end{aligned}
$$

Since we find

$$
\begin{aligned}
-g^{\prime}(t) & =-\frac{(\sigma-1) t^{\sigma-2}}{(x+t)^{s}}+\frac{s t^{\sigma-1}}{(x+t)^{s+1}}=\frac{(1-\sigma) t^{\sigma-2}}{(x+t)^{s}}+\frac{s t^{\sigma-2}}{(x+t)^{s}}-\frac{s x t^{\sigma-2}}{(x+t)^{s+1}} \\
& =\frac{(s+1-\sigma) t^{\sigma-2}}{(x+t)^{s}}-\frac{s x t^{\sigma-2}}{(x+t)^{s+1}},
\end{aligned}
$$

and for $0<\sigma \leq 2,1<s \leq 28$,

$$
(-1)^{i} \frac{d^{i}}{d t^{i}}\left[\frac{t^{\sigma-2}}{(x+t)^{s}}\right]>0, \quad(-1)^{i} \frac{d^{i}}{d t^{i}}\left[\frac{t^{\sigma-2}}{(x+t)^{s+1}}\right]>0 \quad(i=0,1,2,3),
$$


still by the Euler-Maclaurin summation formula (cf. [20]), for $s+1-\sigma>0$, we have

$$
\begin{aligned}
(s+1-\sigma) \int_{1}^{\infty} \rho(t) \frac{t^{\sigma-2}}{(x+t)^{s}} d t>-\frac{s+1-\sigma}{12(x+1)^{s}} \\
-x s \int_{1}^{\infty} \rho(t) \frac{t^{\sigma-2}}{(x+t)^{s+1}} d t \\
>\frac{x s}{12(x+1)^{s+1}}-\frac{x s}{720}\left[\frac{t^{\sigma-2}}{(x+t)^{s+1}}\right]_{t=1}^{\prime \prime} \\
>\frac{(x+1) s-s}{12(x+1)^{s+1}}-\frac{(x+1) s}{720}\left[\frac{(s+1)(s+2)}{(x+1)^{s+3}}+\frac{2(s+1)(2-\sigma)}{(x+1)^{s+2}}+\frac{(2-\sigma)(3-\sigma)}{(x+1)^{s+1}}\right] \\
=\frac{s}{12(x+1)^{s}}-\frac{s}{12(x+1)^{s+1}} \\
\quad-\frac{s}{720}\left[\frac{(s+1)(s+2)}{(x+1)^{s+2}}+\frac{2(s+1)(2-\sigma)}{(x+1)^{s+1}}+\frac{(2-\sigma)(3-\sigma)}{(x+1)^{s}}\right] .
\end{aligned}
$$

Hence, we have $h(\sigma, s)>\frac{h_{1}(\sigma, s)}{(x+1)^{s}}+\frac{s h_{2}(\sigma, s)}{(x+1)^{s+1}}+\frac{s(s+1) h_{3}(\sigma, s)}{(x+1)^{s+2}}$, where

$$
\begin{aligned}
& h_{1}(\sigma, s):=\frac{1}{\sigma}-\frac{1}{2}-\frac{1-\sigma}{12}-\frac{s(2-\sigma)(3-\sigma)}{720}, \\
& h_{2}(\sigma, s):=\frac{1}{\sigma(\sigma+1)}-\frac{1}{12}-\frac{(s+1)(2-\sigma)}{720},
\end{aligned}
$$

and $h_{3}(\sigma, s):=\frac{1}{\sigma(\sigma+1)(\sigma+2)}-\frac{s+2}{720}$.

For $s \in(1,28], \frac{s}{720}<\frac{1}{24}, \sigma \in(0,2]$, it follows that

$$
h_{1}(\sigma, s)>\frac{1}{\sigma}-\frac{1}{2}-\frac{1-\sigma}{12}-\frac{(2-\sigma)(3-\sigma)}{24}=\frac{24-20 \sigma+7 \sigma^{2}-\sigma^{3}}{24 \sigma}>0 .
$$

In fact, setting $g(\sigma):=24-20 \sigma+7 \sigma^{2}-\sigma^{3}(\sigma \in(0,2])$, we obtain

$$
g^{\prime}(\sigma)=-20+14 \sigma^{2}-3 \sigma^{2}=-3\left(\sigma-\frac{7}{3}\right)^{2}-\frac{11}{3}<0
$$

and then $g(\sigma) \geq g(2)=4>0(\sigma \in(0,2])$.

We still find that $h_{2}(\sigma, s)>\frac{1}{6}-\frac{1}{12}-\frac{30}{360}=0$ and $h_{3}(\sigma, s) \geq \frac{1}{24}-\frac{30}{720}=0$. Hence, we have $h(\sigma, s)>0$, and then

$$
\sum_{n=1}^{\infty} g(n)<\int_{0}^{\infty} g(t) d t=\int_{0}^{\infty} \frac{t^{\sigma-1}}{(x+t)^{s}} d t=x^{\sigma-s} \int_{0}^{\infty} \frac{u^{\sigma-1}}{(1+u)^{s}} d u=x^{\sigma-s} B(\sigma, s-\sigma)
$$

namely, (8) follows.

Lemma 3 Suppose that $s \in(1,28], \mu, \sigma \in(1, s), \sigma \in(0,2]$,

$$
0<\int_{0}^{\infty} x^{p\left[1-\left(\frac{s-\sigma}{p}+\frac{\mu}{q}\right)\right]-1} f^{p}(x) d x<\infty \quad \text { and } \quad 0<\sum_{n=1}^{\infty} n^{q\left[1-\left(\frac{\sigma}{p}+\frac{s-\mu}{q}\right)\right]-1} a_{n}^{q}<\infty .
$$


We have the following inequality:

$$
\begin{aligned}
& \int_{0}^{\infty} \sum_{n=1}^{\infty} \frac{f(x) a_{n}}{(x+n)^{s}} d x \\
&<B^{\frac{1}{p}}(\sigma, s-\sigma) B^{\frac{1}{q}}(\mu, s-\mu) \\
& \quad \times\left\{\int_{0}^{\infty} x^{p\left[1-\left(\frac{s-\sigma}{p}+\frac{\mu}{q}\right)\right]-1} f^{p}(x) d x\right\}^{\frac{1}{p}}\left\{\sum_{n=1}^{\infty} n^{q\left[1-\left(\frac{\sigma}{p}+\frac{s-\mu}{q}\right)\right]-1} a_{n}^{q}\right\}^{\frac{1}{q}} .
\end{aligned}
$$

Proof For $n \in \mathbf{N}$, setting $x=n u$, we obtain the following weight function:

$$
\omega(\mu, n):=n^{s-\mu} \int_{0}^{\infty} \frac{x^{\mu-1} d x}{(x+n)^{s}}=\int_{0}^{\infty} \frac{u^{\mu-1} d u}{(u+1)^{s}}=B(\mu, s-\mu) .
$$

By Hölder's inequality (cf. [27]), we obtain

$$
\begin{aligned}
\int_{0}^{\infty} & \sum_{n=1}^{\infty} \frac{f(x) a_{n}}{(x+n)^{s}} d x \\
= & \int_{0}^{\infty} \sum_{n=1}^{\infty} \frac{1}{(x+n)^{s}}\left[\frac{n^{(\sigma-1) / p}}{x^{(\mu-1) / q}} f(x)\right]\left[\frac{x^{(\mu-1) / q}}{n^{(\sigma-1) / p}} a_{n}\right] d x \\
\leq & \left\{\int_{0}^{\infty}\left[\sum_{n=1}^{\infty} \frac{1}{(x+n)^{s}} \frac{n^{\sigma-1}}{x^{(\mu-1)(p-1)}}\right] f^{p}(x) d x\right\}^{\frac{1}{p}} \\
& \times\left\{\sum_{n=1}^{\infty}\left[\int_{0}^{\infty} \frac{1}{(x+n)^{s}} \frac{x^{\mu-1}}{n^{(\sigma-1)(q-1)}} d x\right] a_{n}^{q}\right\}^{\frac{1}{q}} \\
= & \left\{\int_{0}^{\infty} \varpi(\sigma, x) x^{p\left[1-\left(\frac{s-\sigma}{p}+\frac{\mu}{q}\right)\right]-1} f^{p}(x) d x\right\}^{\frac{1}{p}}\left\{\sum_{n=1}^{\infty} \omega(\mu, n) n^{q\left[1-\left(\frac{\sigma}{p}+\frac{s-\mu}{q}\right)\right]-1} a_{n}^{q}\right\}^{\frac{1}{q}} .
\end{aligned}
$$

Then, by (8) and (10), we have (9).

Remark 1 For $s=\lambda+2, \lambda \in(-1,26], \lambda_{1}=\mu-1 \in(0, \lambda+1), \lambda_{2}=\sigma-1 \in(0,1] \cap(0, \lambda+1)$, we can reduce (9) as follows:

$$
\begin{aligned}
\int_{0}^{\infty} & \sum_{n=1}^{\infty} \frac{F(x) A_{n}}{(x+n)^{\lambda+2}} d x \\
< & B^{\frac{1}{p}}\left(\lambda_{2}+1, \lambda+1-\lambda_{2}\right) B^{\frac{1}{q}}\left(\lambda_{1}+1, \lambda+1-\lambda_{1}\right) \\
& \times\left\{\int_{0}^{\infty} x^{p\left[1-\left(\frac{\lambda+1-\lambda_{2}}{p}+\frac{\lambda_{1}+1}{q}\right)\right]-1} F^{p}(x) d x\right\}^{\frac{1}{p}}\left\{\sum_{n=1}^{\infty} n^{q\left[1-\left(\frac{\lambda_{2}+1}{p}+\frac{\lambda+1-\lambda_{1}}{q}\right)\right]-1} A_{n}^{q}\right\}^{\frac{1}{q}} .
\end{aligned}
$$




\section{Main results}

Theorem 1 If $\lambda \in(0,26], \lambda_{1}, \lambda_{2} \in(0, \lambda+1), \lambda_{2} \in(0,1]$, then we have the following inequality:

$$
\begin{aligned}
I:= & \int_{0}^{\infty} \sum_{n=1}^{\infty} \frac{f(x) a_{n}}{(x+n)^{\lambda}} d x \\
< & \frac{\Gamma(\lambda+2)}{\Gamma(\lambda)} B^{\frac{1}{p}}\left(\lambda_{2}+1, \lambda+1-\lambda_{2}\right) B^{\frac{1}{q}}\left(\lambda_{1}+1, \lambda+1-\lambda_{1}\right) \\
& \times\left\{\int_{0}^{\infty} x^{p\left[1-\left(\frac{\lambda+1-\lambda_{2}}{p}+\frac{\lambda_{1}+1}{q}\right)\right]-1} F^{p}(x) d x\right\}^{\frac{1}{p}}\left\{\sum_{n=1}^{\infty} n^{q\left[1-\left(\frac{\lambda_{2}+1}{p}+\frac{\lambda+1-\lambda_{1}}{q}\right)\right]-1} A_{n}^{q}\right\}^{\frac{1}{q}} .
\end{aligned}
$$

In particular, for $\lambda_{1}+\lambda_{2}=\lambda$, we also have

$$
\int_{0}^{\infty} \sum_{n=1}^{\infty} \frac{f(x) a_{n}}{(x+n)^{\lambda}} d x<\lambda_{1} \lambda_{2} B\left(\lambda_{1}, \lambda_{2}\right)\left(\int_{0}^{\infty} x^{-p \lambda_{1}-1} F^{p}(x) d x\right)^{\frac{1}{p}}\left(\sum_{n=1}^{\infty} n^{-q \lambda_{2}-1} A_{n}^{q}\right)^{\frac{1}{q}},
$$

where the constant factor $\lambda_{1} \lambda_{2} B\left(\lambda_{1}, \lambda_{2}\right)$ is the best possible.

Proof Using (4), (5), and (6), we find

$$
\begin{aligned}
I & =\frac{1}{\Gamma(\lambda)} \int_{0}^{\infty} \sum_{n=1}^{\infty} a_{n} f(x)\left(\int_{0}^{\infty} t^{\lambda-1} e^{-(x+n) t} d t\right) d x \\
& =\frac{1}{\Gamma(\lambda)} \int_{0}^{\infty} t^{\lambda-1}\left(\int_{0}^{\infty} e^{-x t} f(x) d x\right)\left(\sum_{n=1}^{\infty} e^{-n t} a_{n}\right) d t \\
& \leq \frac{1}{\Gamma(\lambda)} \int_{0}^{\infty} t^{\lambda+1}\left(\int_{0}^{\infty} e^{-x t} F(x) d x\right)\left(\sum_{n=1}^{\infty} e^{-n t} A_{n}\right) d t \\
& =\frac{1}{\Gamma(\lambda)} \int_{0}^{\infty} \sum_{n=1}^{\infty} F(x) A_{n}\left(\int_{0}^{\infty} t^{\lambda+1} e^{-(x+n) t} d t\right) d x \\
& =\frac{\Gamma(\lambda+2)}{\Gamma(\lambda)} \int_{0}^{\infty} \sum_{n=1}^{\infty} \frac{F(x) A_{n}}{(x+n)^{\lambda+2}} d x .
\end{aligned}
$$

In view of (11), we have (12).

In the case of $\lambda_{1}+\lambda_{2}=\lambda$, we find

$$
\begin{aligned}
& \frac{\Gamma(\lambda+2)}{\Gamma(\lambda)} B^{\frac{1}{p}}\left(\lambda_{2}+1, \lambda+1-\lambda_{2}\right) B^{\frac{1}{q}}\left(\lambda_{1}+1, \lambda+1-\lambda_{1}\right) \\
& \quad=\frac{\Gamma(\lambda+2)}{\Gamma(\lambda)} B^{\frac{1}{p}}\left(\lambda_{2}+1, \lambda_{1}+1\right) B^{\frac{1}{q}}\left(\lambda_{1}+1, \lambda_{2}+1\right)=\frac{\Gamma(\lambda+2)}{\Gamma(\lambda)} B\left(\lambda_{1}+1, \lambda_{2}+1\right) \\
& \quad=\frac{\Gamma(\lambda+2)}{\Gamma(\lambda)} \frac{\Gamma\left(\lambda_{1}+1\right) \Gamma\left(\lambda_{2}+1\right)}{\Gamma(\lambda+2)}=\lambda_{1} \lambda_{2} \frac{\Gamma\left(\lambda_{1}\right) \Gamma\left(\lambda_{2}\right)}{\Gamma(\lambda)}=\lambda_{1} \lambda_{2} B\left(\lambda_{1}, \lambda_{2}\right),
\end{aligned}
$$

and then (13) follows. 
For any $0<\varepsilon<\min \left\{p \lambda_{1}, q \lambda_{2}\right\}$, we set

$$
\tilde{f}(t):=\left\{\begin{array}{ll}
0, & 0<t<1, \\
t^{\lambda_{1}-\frac{\varepsilon}{p}-1}, & t \geq 1
\end{array}, \quad \tilde{a}_{k}:=k^{\lambda_{2}-\frac{\varepsilon}{q}-1} \quad(k \in \mathbf{N}) .\right.
$$

We obtain from $\lambda_{1}, \lambda_{2} \in(0, \lambda+1), \lambda_{2} \in(0,1]$, and $0<\varepsilon<\min \left\{p \lambda_{1}, q \lambda_{2}\right\}$ that $\tilde{F}(x)=0(0<$ $x<1)$,

$$
\begin{aligned}
& \tilde{F}(x)=\int_{0}^{x} \tilde{f}(t) d t=\int_{1}^{x} t^{\lambda_{1}-\frac{\varepsilon}{p}-1} d t \leq \frac{x^{\lambda_{1}-\frac{\varepsilon}{p}}}{\lambda_{1}-\frac{\varepsilon}{p}} \quad(x \geq 1), \\
& \tilde{A}_{n}:=\sum_{k=1}^{n} \tilde{a}_{k}=\sum_{k=1}^{n} k^{\lambda_{2}-\frac{\varepsilon}{q}-1}<\int_{0}^{n} t^{\lambda_{2}-\frac{\varepsilon}{q}-1} d t=\frac{n^{\lambda_{2}-\frac{\varepsilon}{q}}}{\lambda_{2}-\frac{\varepsilon}{q}} \quad(n \in \mathbf{N}) .
\end{aligned}
$$

If there exists a positive constant $M\left(M \leq \lambda_{1} \lambda_{2} B\left(\lambda_{1}, \lambda_{2}\right)\right)$ such that (13) is valid when replacing $\lambda_{1} \lambda_{2} B\left(\lambda_{1}, \lambda_{2}\right)$ by $M$, then in particular, by substitution of $f(x)=\tilde{f}(x)$ and $a_{n}=\tilde{a}_{n}$, we have

$$
\tilde{I}:=\int_{0}^{\infty} \sum_{n=1}^{\infty} \frac{\tilde{f}(x) \tilde{a}_{n}}{(x+n)^{\lambda}} d x<M\left(\int_{0}^{\infty} x^{-p \lambda_{1}-1} \tilde{F}^{p}(x) d x\right)^{\frac{1}{p}}\left(\sum_{n=1}^{\infty} n^{-q \lambda_{2}-1} \tilde{A}_{n}^{q}\right)^{\frac{1}{q}} .
$$

We find

$$
\begin{aligned}
\tilde{J} & :=\left(\int_{0}^{\infty} x^{-p \lambda_{1}-1} \tilde{F}^{p}(x) d x\right)^{\frac{1}{p}}\left(\sum_{n=1}^{\infty} n^{-q \lambda_{2}-1} \tilde{A}_{n}^{q}\right)^{\frac{1}{q}} \\
& <\frac{1}{\left(\lambda_{1}-\frac{\varepsilon}{p}\right)\left(\lambda_{2}-\frac{\varepsilon}{q}\right)}\left[\int_{1}^{\infty} x^{-p \lambda_{1}-1}\left(x^{\lambda_{1}-\frac{\varepsilon}{p}}\right)^{p} d x\right]^{\frac{1}{p}}\left[\sum_{n=1}^{\infty} n^{-q \lambda_{2}-1}\left(n^{\lambda_{2}-\frac{\varepsilon}{q}}\right)^{q}\right]^{\frac{1}{q}} \\
& =\frac{1}{\left(\lambda_{1}-\frac{\varepsilon}{p}\right)\left(\lambda_{2}-\frac{\varepsilon}{q}\right)}\left(\int_{1}^{\infty} x^{-\varepsilon-1} d x\right)^{\frac{1}{p}}\left(1+\sum_{n=2}^{\infty} n^{-\varepsilon-1}\right)^{\frac{1}{q}} \\
& <\frac{1}{\left(\lambda_{1}-\frac{\varepsilon}{p}\right)\left(\lambda_{2}-\frac{\varepsilon}{q}\right)}\left(\int_{1}^{\infty} x^{-\varepsilon-1} d x\right)^{\frac{1}{p}}\left(1+\int_{1}^{\infty} t^{-\varepsilon-1} d t\right)^{\frac{1}{q}}=\frac{(\varepsilon+1)^{1 / q}}{\varepsilon\left(\lambda_{1}-\frac{\varepsilon}{p}\right)\left(\lambda_{2}-\frac{\varepsilon}{q}\right)} .
\end{aligned}
$$

In view of Fubini's theorem (cf. [28]), it follows that

$$
\begin{aligned}
\tilde{I} & =\int_{1}^{\infty} \sum_{n=1}^{\infty} \frac{n^{\lambda_{2}-\frac{\varepsilon}{q}-1}}{(x+n)^{\lambda}} x^{\lambda_{1}-\frac{\varepsilon}{p}-1} d x \geq \int_{1}^{\infty}\left(\int_{1}^{\infty} \frac{t^{\lambda_{2}-\frac{\varepsilon}{q}-1}}{(x+t)^{\lambda}} d t\right) x^{\lambda_{1}-\frac{\varepsilon}{p}-1} d x \\
& =\int_{1}^{\infty} x^{-\varepsilon-1} \int_{1 / x}^{\infty} \frac{u^{\lambda_{2}-\frac{\varepsilon}{q}-1}}{(1+u)^{\lambda}} d u d x \\
& =\int_{1}^{\infty} x^{-\varepsilon-1} \int_{1 / x}^{1} \frac{u^{\lambda_{2}-\frac{\varepsilon}{q}-1}}{(1+u)^{\lambda}} d u d x+\int_{1}^{\infty} x^{-\varepsilon-1} \int_{1}^{\infty} \frac{u^{\lambda_{2}-\frac{\varepsilon}{q}-1}}{(1+u)^{\lambda}} d u d x
\end{aligned}
$$




$$
\begin{aligned}
& =\int_{0}^{1}\left(\int_{1 / u}^{\infty} x^{-\varepsilon-1} d x\right) \frac{u^{\lambda_{2}-\frac{\varepsilon}{q}-1}}{(1+u)^{\lambda}} d u+\frac{1}{\varepsilon} \int_{1}^{\infty} \frac{u^{\lambda_{2}-\frac{\varepsilon}{q}-1}}{(1+u)^{\lambda}} d u \\
& =\frac{1}{\varepsilon}\left[\int_{0}^{1} \frac{u^{\lambda_{2}+\frac{\varepsilon}{p}-1}}{(1+u)^{\lambda}} d u+\int_{1}^{\infty} \frac{u^{\lambda_{2}-\frac{\varepsilon}{q}-1}}{(1+u)^{\lambda}} d u\right] .
\end{aligned}
$$

So we obtain

$$
\int_{0}^{1} \frac{u^{\lambda_{2}+\frac{\varepsilon}{p}-1}}{(1+u)^{\lambda}} d u+\int_{1}^{\infty} \frac{u^{\lambda_{2}-\frac{\varepsilon}{q}-1}}{(1+u)^{\lambda}} d u \leq \varepsilon \tilde{I}<\varepsilon M \tilde{J}<\frac{M(\varepsilon+1)^{1 / q}}{\left(\lambda_{1}-\frac{\varepsilon}{p}\right)\left(\lambda_{2}-\frac{\varepsilon}{q}\right)}
$$

For $\varepsilon \rightarrow 0^{+}$in the above inequality, in view of the continuity of the beta function, we find $B\left(\lambda_{1}, \lambda_{2}\right) \leq \frac{M}{\lambda_{1} \lambda_{2}}$, namely $\lambda_{1} \lambda_{2} B\left(\lambda_{1}, \lambda_{2}\right) \leq M$. Hence $M=\lambda_{1} \lambda_{2} B\left(\lambda_{1}, \lambda_{2}\right)$ is the best possible constant factor of (13).

Remark 2 We set $\hat{\lambda}_{1}:=\frac{\lambda+1-\lambda_{2}}{p}+\frac{\lambda_{1}+1}{q}-1, \hat{\lambda}_{2}:=\frac{\lambda_{2}+1}{p}+\frac{\lambda+1-\lambda_{1}}{q}-1$. It follows that

$$
\hat{\lambda}_{1}+\hat{\lambda}_{2}=\frac{\lambda+1-\lambda_{2}}{p}+\frac{\lambda_{1}+1}{q}-1+\frac{\lambda_{2}+1}{p}+\frac{\lambda+1-\lambda_{1}}{q}-1=\lambda,
$$

$0<\hat{\lambda}_{1}, \hat{\lambda}_{2}<\lambda+1$, and then we reduce (12) as follows:

$$
\begin{aligned}
I:= & \int_{0}^{\infty} \sum_{n=1}^{\infty} \frac{f(x) a_{n}}{(x+n)^{\lambda}} d x \\
< & \frac{\Gamma(\lambda+2)}{\Gamma(\lambda)} B^{\frac{1}{p}}\left(\lambda_{2}+1, \lambda+1-\lambda_{2}\right) B^{\frac{1}{q}}\left(\lambda_{1}+1, \lambda+1-\lambda_{1}\right) \\
& \times\left(\int_{0}^{\infty} x^{-p \hat{\lambda}_{1}-1} F^{p}(x) d x\right)^{\frac{1}{p}}\left(\sum_{n=1}^{\infty} n^{-q \hat{\lambda}_{2}-1} A_{n}^{q}\right)^{\frac{1}{q}}
\end{aligned}
$$

Theorem 2 Assuming that $\lambda \in(0,26], \lambda_{1}, \lambda_{2} \in(0, \lambda+1), \lambda_{2} \in(0,1]$, if the constant factor

$$
\frac{\Gamma(\lambda+2)}{\Gamma(\lambda)} B^{\frac{1}{p}}\left(\lambda_{2}+1, \lambda+1-\lambda_{2}\right) B^{\frac{1}{q}}\left(\lambda_{1}+1, \lambda+1-\lambda_{1}\right)
$$

in (15) is the best possible, then $\lambda_{1}+\lambda_{2}=\lambda$.

Proof As regards to the assumptions, we find $0<\hat{\lambda}_{1}, \hat{\lambda}_{2}<\lambda+1$. By (13), the unified best possible constant factor in (15) must be of the following form:

$$
\hat{\lambda}_{1} \hat{\lambda}_{2} B\left(\hat{\lambda}_{1}, \hat{\lambda}_{2}\right)\left(=\frac{\Gamma(\lambda+2)}{\Gamma(\lambda)} B\left(\hat{\lambda}_{1}+1, \hat{\lambda}_{2}+1\right)\right)
$$

namely, it follows that

$$
B\left(\hat{\lambda}_{1}+1, \hat{\lambda}_{2}+1\right)=B^{\frac{1}{p}}\left(\lambda_{2}+1, \lambda+1-\lambda_{2}\right) B^{\frac{1}{q}}\left(\lambda_{1}+1, \lambda+1-\lambda_{1}\right) .
$$


By Hölder's inequality (cf. [27]), we obtain

$$
\begin{aligned}
B\left(\hat{\lambda}_{1}+1, \hat{\lambda}_{2}+1\right) & =\int_{0}^{\infty} \frac{u^{\left(\hat{\lambda}_{1}+1\right)-1}}{(1+u)^{\lambda+2}} d u=\int_{0}^{\infty} \frac{u^{\hat{\lambda}_{1}}}{(1+u)^{\lambda+2}} d u \\
& =\int_{0}^{\infty} \frac{1}{(1+u)^{\lambda+2}} u^{\frac{\lambda+1-\lambda_{2}}{p}+\frac{\lambda_{1}+1}{q}-1} d u=\int_{0}^{\infty} \frac{1}{(1+u)^{\lambda+2}}\left(u^{\frac{\lambda-\lambda_{2}}{p}}\right)\left(u^{\frac{\lambda_{1}}{q}}\right) d u \\
& \leq\left\{\int_{0}^{\infty} \frac{u^{\lambda-\lambda_{2}}}{(1+u)^{\lambda+2}} d u\right\}^{\frac{1}{p}}\left\{\int_{0}^{\infty} \frac{u^{\lambda_{1}}}{(1+u)^{\lambda+2}} d u\right\}^{\frac{1}{q}} \\
& =B^{\frac{1}{p}}\left(\lambda_{2}+1, \lambda+1-\lambda_{2}\right) B^{\frac{1}{q}}\left(\lambda_{1}+1, \lambda+1-\lambda_{1}\right) .
\end{aligned}
$$

We observe that (16) keeps the form of equality if and only if there exist constants $A$ and $B$ such that they are not all zero and $A u^{\lambda-\lambda_{2}}=B u^{\lambda_{1}}$ a.e. in $R_{+}$. Assuming that $A \neq 0$, it follows that $u^{\lambda-\lambda_{2}-\lambda_{1}}=\frac{B}{A}$ a.e. in $R_{+}$, namely $\lambda-\lambda_{2}-\lambda_{1}=0$, and then $\lambda_{1}+\lambda_{2}=\lambda$.

Theorem 3 If $\lambda \in(0,26], \lambda_{1}, \lambda_{2} \in(0, \lambda+1), \lambda_{2} \in(0,1]$, then the following statements are equivalent:

(i) $B^{\frac{1}{p}}\left(\lambda_{2}+1, \lambda+1-\lambda_{2}\right) B^{\frac{1}{q}}\left(\lambda_{1}+1, \lambda+1-\lambda_{1}\right)$ is independent of $p, q$;

(ii) $B^{\frac{1}{p}}\left(\lambda_{2}+1, \lambda+1-\lambda_{2}\right) B^{\frac{1}{q}}\left(\lambda_{1}+1, \lambda+1-\lambda_{1}\right)$ is expressible as a single integral;

(iii) $\lambda_{1}+\lambda_{2}=\lambda$;

(iv) The constant factor

$$
\frac{\Gamma(\lambda+2)}{\Gamma(\lambda)} B^{\frac{1}{p}}\left(\lambda_{2}+1, \lambda+1-\lambda_{2}\right) B^{\frac{1}{q}}\left(\lambda_{1}+1, \lambda+1-\lambda_{1}\right)
$$

in (12) is the best possible.

Proof (i) $\Rightarrow$ (ii). We find

$$
\begin{aligned}
& B^{\frac{1}{p}}\left(\lambda_{2}+1, \lambda+1-\lambda_{2}\right) B^{\frac{1}{q}}\left(\lambda_{1}+1, \lambda+1-\lambda_{1}\right) \\
& \quad=\lim _{p \rightarrow \infty} \lim _{q \rightarrow 1^{+}} B^{\frac{1}{p}}\left(\lambda_{2}+1, \lambda+1-\lambda_{2}\right) B^{\frac{1}{q}}\left(\lambda_{1}+1, \lambda+1-\lambda_{1}\right) \\
& \quad=B\left(\lambda_{1}+1, \lambda+1-\lambda_{1}\right)=\int_{0}^{\infty} \frac{u^{\lambda_{1}}}{(1+u)^{\lambda+2}} d u
\end{aligned}
$$

which is a single integral. (ii) $\Rightarrow$ (iii). Suppose that $B^{\frac{1}{p}}\left(\lambda_{2}+1, \lambda+1-\lambda_{2}\right) B^{\frac{1}{q}}\left(\lambda_{1}+1, \lambda+1-\lambda_{1}\right)$ is expressible as a single integral $\int_{0}^{\infty} \frac{1}{(1+u)^{\lambda+2}} u^{\frac{\lambda+1-\lambda_{2}}{p}+\frac{\lambda_{1}+1}{q}-1} d u$. Then (16) keeps the form of equality. By the proof of Theorem 2 , we have $\lambda_{1}+\lambda_{2}=\lambda$. (iii) $\Rightarrow$ (i). If $\lambda_{1}+\lambda_{2}=\lambda$, then

$$
B^{\frac{1}{p}}\left(\lambda_{2}+1, \lambda+1-\lambda_{2}\right) B^{\frac{1}{q}}\left(\lambda_{1}+1, \lambda+1-\lambda_{1}\right)=B\left(\lambda_{1}+1, \lambda_{2}+1\right),
$$

which is a single integral.

(iii) $\Rightarrow$ (iv). By Theorem 1 , for $\lambda_{1}+\lambda_{2}=\lambda$, the constant factor

$$
\frac{\Gamma(\lambda+2)}{\Gamma(\lambda)} B^{\frac{1}{p}}\left(\lambda_{2}+1, \lambda+1-\lambda_{2}\right) B^{\frac{1}{q}}\left(\lambda_{1}+1, \lambda+1-\lambda_{1}\right)=\lambda_{1} \lambda_{2} B\left(\lambda_{1}, \lambda_{2}\right)
$$

in (12) is the best possible. (iv) $\Rightarrow$ (iii). By Theorem 2 , we have $\lambda_{1}+\lambda_{2}=\lambda$. 
Hence, statements (i), (ii), (iii), and (iv) are equivalent.

Remark 3 If $\mu+\sigma=s$, then inequality (9) reduces to

$$
\int_{0}^{\infty} \sum_{n=1}^{\infty} \frac{f(x) a_{n}}{(x+n)^{s}} d x<B(\mu, \sigma)\left[\int_{0}^{\infty} x^{p(1-\mu)-1} f^{p}(x) d x\right]^{\frac{1}{p}}\left[\sum_{n=1}^{\infty} n^{q(1-\sigma)-1} a_{n}^{q}\right]^{\frac{1}{q}}
$$

We confirm that the constant factor $B(\mu, \sigma)$ in (17) is the best possible. Otherwise, we would reach a contradiction by (14) that the constant factor in (13) is not the best possible.

\section{A corollary and some particular cases}

Replacing $x$ by $\frac{1}{x}$ in (12), setting $g(x)=x^{\lambda-2} f\left(\frac{1}{x}\right)$, we define

$$
G_{\lambda}(x):=F(x)=\int_{0}^{x} f(t) d t=\int_{\frac{1}{x}}^{\infty} f\left(\frac{1}{u}\right) \frac{1}{u^{2}} d u=\int_{\frac{1}{x}}^{\infty} t^{-\lambda} g(t) d t
$$

Then we obtain the following inequality with the non-homogeneous kernel:

$$
\begin{aligned}
\int_{0}^{\infty} & \sum_{n=1}^{\infty} \frac{g(x) a_{n}}{(1+x n)^{\lambda}} d x \\
< & \frac{\Gamma(\lambda+2)}{\Gamma(\lambda)} B^{\frac{1}{p}}\left(\lambda_{2}+1, \lambda+1-\lambda_{2}\right) B^{\frac{1}{q}}\left(\lambda_{1}+1, \lambda+1-\lambda_{1}\right) \\
& \times\left\{\int_{0}^{\infty} x^{p\left[1-\left(\frac{\lambda+1-\lambda_{2}}{p}+\frac{\lambda_{1}+1}{q}\right)\right]-1} G_{\lambda}^{p}(x) d x\right\}^{\frac{1}{p}}\left\{\sum_{n=1}^{\infty} n^{q\left[1-\left(\frac{\lambda_{2}+1}{p}+\frac{\lambda+1-\lambda_{1}}{q}\right)\right]-1} A_{n}^{q}\right\}^{\frac{1}{q}}
\end{aligned}
$$

It is obvious that inequality (18) is equivalent to (12).

In view of Theorem 3 , we have the following.

Corollary 1 Assuming that $\lambda \in(0,26], \lambda_{1}, \lambda_{2} \in(0, \lambda+1), \lambda_{2} \in(0,1]$, the constant factor

$$
\frac{\Gamma(\lambda+2)}{\Gamma(\lambda)} B^{\frac{1}{p}}\left(\lambda_{2}+1, \lambda+1-\lambda_{2}\right) B^{\frac{1}{q}}\left(\lambda_{1}+1, \lambda+1-\lambda_{1}\right)
$$

in (18) is the best possible if and only if $\lambda_{1}+\lambda_{2}=\lambda$. In the case of $\lambda_{1}+\lambda_{2}=\lambda$, (18) reduces to the following inequality with the best possible constant factor $\lambda_{1} \lambda_{2} B\left(\lambda_{1}, \lambda_{2}\right)$ :

$$
\begin{aligned}
& \int_{0}^{\infty} \sum_{n=1}^{\infty} \frac{g(x) a_{n}}{(1+x n)^{\lambda}} d x \\
& \quad<\lambda_{1} \lambda_{2} B\left(\lambda_{1}, \lambda_{2}\right)\left(\int_{0}^{\infty} x^{-p \lambda_{1}-1} G_{\lambda}^{p}(x) d x\right)^{\frac{1}{p}}\left(\sum_{n=1}^{\infty} n^{-q \lambda_{2}-1} A_{n}^{q}\right)^{\frac{1}{q}},
\end{aligned}
$$

which is equivalent to (13). 
Remark 4 (i) In (13) and (19), for $0<\lambda \leq \min \{p, 26\}, \lambda_{1}=\frac{\lambda}{q}, \lambda_{2}=\frac{\lambda}{p}(\leq 1)$, we have the following equivalent inequalities:

$$
\begin{aligned}
& \int_{0}^{\infty} \sum_{n=1}^{\infty} \frac{f(x) a_{n}}{(x+n)^{\lambda}} d x \\
&< \frac{\lambda^{2}}{p q} B\left(\frac{\lambda}{p}, \frac{\lambda}{q}\right)\left(\int_{0}^{\infty} x^{\lambda(1-p)-1} F^{p}(x) d x\right)^{\frac{1}{p}}\left(\sum_{n=1}^{\infty} n^{\lambda(1-q)-1} A_{n}^{q}\right)^{\frac{1}{q}}, \\
& \int_{0}^{\infty} \sum_{n=1}^{\infty} \frac{g(x) a_{n}}{(1+x n)^{\lambda}} d x \\
&<\frac{\lambda^{2}}{p q} B\left(\frac{\lambda}{p}, \frac{\lambda}{q}\right)\left(\int_{0}^{\infty} x^{\lambda(1-p)-1} G_{\lambda}^{p}(x) d x\right)^{\frac{1}{p}}\left(\sum_{n=1}^{\infty} n^{\lambda(1-q)-1} A_{n}^{q}\right)^{\frac{1}{q}} ;
\end{aligned}
$$

if $0<\lambda \leq \min \{q, 26\}, \lambda_{1}=\frac{\lambda}{p}, \lambda_{2}=\frac{\lambda}{q}(\leq 1)$, then we have the following equivalent inequalities:

$$
\begin{aligned}
& \int_{0}^{\infty} \sum_{n=1}^{\infty} \frac{f(x) a_{n}}{(x+n)^{\lambda}} d x<\frac{\lambda^{2}}{p q} B\left(\frac{\lambda}{p}, \frac{\lambda}{q}\right)\left(\int_{0}^{\infty} x^{-\lambda-1} F^{p}(x) d x\right)^{\frac{1}{p}}\left(\sum_{n=1}^{\infty} n^{-\lambda-1} A_{n}^{q}\right)^{\frac{1}{q}}, \\
& \int_{0}^{\infty} \sum_{n=1}^{\infty} \frac{g(x) a_{n}}{(1+x n)^{\lambda}} d x<\frac{\lambda^{2}}{p q} B\left(\frac{\lambda}{p}, \frac{\lambda}{q}\right)\left(\int_{0}^{\infty} x^{-\lambda-1} G_{\lambda}^{p}(x) d x\right)^{\frac{1}{p}}\left(\sum_{n=1}^{\infty} n^{-\lambda-1} A_{n}^{q}\right)^{\frac{1}{q}} .
\end{aligned}
$$

In particular, for $p=q=2,0<\lambda \leq 2$, both inequalities (20) and (22) reduce to

$$
\int_{0}^{\infty} \sum_{n=1}^{\infty} \frac{f(x) a_{n}}{(x+n)^{\lambda}} d x<\frac{\lambda^{2}}{4} B\left(\frac{\lambda}{2}, \frac{\lambda}{2}\right)\left(\int_{0}^{\infty} x^{-\lambda-1} F^{2}(x) d x \sum_{n=1}^{\infty} n^{-\lambda-1} A_{n}^{2}\right)^{\frac{1}{2}},
$$

and both (21) and (23) reduce to the equivalent form of (24) as follows:

$$
\int_{0}^{\infty} \sum_{n=1}^{\infty} \frac{g(x) a_{n}}{(1+x n)^{\lambda}} d x<\frac{\lambda^{2}}{4} B\left(\frac{\lambda}{2}, \frac{\lambda}{2}\right)\left(\int_{0}^{\infty} x^{-\lambda-1} G_{\lambda}^{2}(x) d x \sum_{n=1}^{\infty} n^{-\lambda-1} A_{n}^{2}\right)^{\frac{1}{2}} .
$$

(ii) In (13) and (19), for $\frac{1}{p}<\lambda \leq 26, \lambda_{1}=\lambda-\frac{1}{p}, \lambda_{2}=\frac{1}{p}(<1)$, we have the following equivalent inequalities:

$$
\begin{aligned}
& \int_{0}^{\infty} \sum_{n=1}^{\infty} \frac{f(x) a_{n}}{(x+n)^{\lambda}} d x \\
& \quad<\frac{p \lambda-1}{p^{2}} B\left(\frac{p \lambda-1}{p}, \frac{1}{p}\right)\left(\int_{0}^{\infty} x^{-p \lambda} F^{p}(x) d x\right)^{\frac{1}{p}}\left(\sum_{n=1}^{\infty} n^{-q} A_{n}^{q}\right)^{\frac{1}{q}}, \\
& \int_{0}^{\infty} \sum_{n=1}^{\infty} \frac{g(x) a_{n}}{(1+x n)^{\lambda}} d x \\
& <\frac{p \lambda-1}{p^{2}} B\left(\frac{p \lambda-1}{p}, \frac{1}{p}\right)\left(\int_{0}^{\infty} x^{-p \lambda} G_{\lambda}^{p}(x) d x\right)^{\frac{1}{p}}\left(\sum_{n=1}^{\infty} n^{-q} A_{n}^{q}\right)^{\frac{1}{q}} ;
\end{aligned}
$$


if $\frac{1}{q}<\lambda \leq 26, \lambda_{1}=\lambda-\frac{1}{q}, \lambda_{2}=\frac{1}{q}(<1)$, then we have the following equivalent inequalities:

$$
\begin{aligned}
& \int_{0}^{\infty} \sum_{n=1}^{\infty} \frac{f(x) a_{n}}{(x+n)^{\lambda}} d x \\
& <\frac{q \lambda-1}{q^{2}} B\left(\frac{q \lambda-1}{q}, \frac{1}{q}\right)\left(\int_{0}^{\infty} x^{-2 \lambda} F^{p}(x) d x\right)^{\frac{1}{p}}\left(\sum_{n=1}^{\infty} n^{-2} A_{n}^{q}\right)^{\frac{1}{q}}, \\
& \int_{0}^{\infty} \sum_{n=1}^{\infty} \frac{g(x) a_{n}}{(1+x n)^{\lambda}} d x \\
& <\frac{q \lambda-1}{q^{2}} B\left(\frac{q \lambda-1}{q}, \frac{1}{q}\right)\left(\int_{0}^{\infty} x^{-2 \lambda} G_{\lambda}^{p}(x) d x\right)^{\frac{1}{p}}\left(\sum_{n=1}^{\infty} n^{-2} A_{n}^{q}\right)^{\frac{1}{q}} .
\end{aligned}
$$

In particular, for $p=q=2, \frac{1}{2}<\lambda \leq 26$, both inequalities (26) and (28) reduce to

$$
\int_{0}^{\infty} \sum_{n=1}^{\infty} \frac{f(x) a_{n}}{(x+n)^{\lambda}} d x<\frac{2 \lambda-1}{4} B\left(\frac{2 \lambda-1}{2}, \frac{1}{2}\right)\left(\int_{0}^{\infty} x^{-2 \lambda} F^{2}(x) d x \sum_{n=1}^{\infty} n^{-2} A_{n}^{2}\right)^{\frac{1}{2}}
$$

and both (27) and (29) reduce to the equivalent form of (30) as follows:

$$
\int_{0}^{\infty} \sum_{n=1}^{\infty} \frac{g(x) a_{n}}{(1+x n)^{\lambda}} d x<\frac{2 \lambda-1}{4} B\left(\frac{2 \lambda-1}{2}, \frac{1}{2}\right)\left(\int_{0}^{\infty} x^{-2 \lambda} G_{\lambda}^{2}(x) d x \sum_{n=1}^{\infty} n^{-2} A_{n}^{2}\right)^{\frac{1}{2}} .
$$

(iii) In (13) and (19), for $1<\lambda \leq 26, \lambda_{1}=\lambda-1, \lambda_{2}=1$, we have the following equivalent inequalities:

$$
\begin{aligned}
& \int_{0}^{\infty} \sum_{n=1}^{\infty} \frac{f(x) a_{n}}{(x+n)^{\lambda}} d x<\left(\int_{0}^{\infty} x^{p(1-\lambda)-1} F^{p}(x) d x\right)^{\frac{1}{p}}\left(\sum_{n=1}^{\infty} n^{-q-1} A_{n}^{q}\right)^{\frac{1}{q}}, \\
& \int_{0}^{\infty} \sum_{n=1}^{\infty} \frac{g(x) a_{n}}{(1+x n)^{\lambda}} d x\left(\int_{0}^{\infty} x^{p(1-\lambda)-1} G_{\lambda}^{p}(x) d x\right)^{\frac{1}{p}}\left(\sum_{n=1}^{\infty} n^{-q-1} A_{n}^{q}\right)^{\frac{1}{q}}
\end{aligned}
$$

if $1<\lambda \leq 2, \lambda_{1}=1, \lambda_{2}=\lambda-1(\leq 1)$, we have the following equivalent inequalities:

$$
\begin{aligned}
& \int_{0}^{\infty} \sum_{n=1}^{\infty} \frac{f(x) a_{n}}{(x+n)^{\lambda}} d x<\left(\int_{0}^{\infty} x^{-p-1} F^{p}(x) d x\right)^{\frac{1}{p}}\left(\sum_{n=1}^{\infty} n^{q(1-\lambda)-1} A_{n}^{q}\right)^{\frac{1}{q}}, \\
& \int_{0}^{\infty} \sum_{n=1}^{\infty} \frac{g(x) a_{n}}{(1+x n)^{\lambda}} d x\left(\int_{0}^{\infty} x^{-p-1} G_{\lambda}^{p}(x) d x\right)^{\frac{1}{p}}\left(\sum_{n=1}^{\infty} n^{q(1-\lambda)-1} A_{n}^{q}\right)^{\frac{1}{q}} .
\end{aligned}
$$

In particular, for $\lambda=2$, both (32) and (34) reduce to

$$
\int_{0}^{\infty} \sum_{n=1}^{\infty} \frac{f(x) a_{n}}{(x+n)^{2}} d x<\left(\int_{0}^{\infty} x^{-p-1} F^{p}(x) d x\right)^{\frac{1}{p}}\left(\sum_{n=1}^{\infty} n^{-q-1} A_{n}^{q}\right)^{\frac{1}{q}},
$$


both (33) and (35) reduce to the equivalent form of (36) as follows:

$$
\int_{0}^{\infty} \sum_{n=1}^{\infty} \frac{g(x) a_{n}}{(1+x n)^{2}} d x\left(\int_{0}^{\infty} x^{-p-1} G_{2}^{p}(x) d x\right)^{\frac{1}{p}}\left(\sum_{n=1}^{\infty} n^{-q-1} A_{n}^{q}\right)^{\frac{1}{q}}
$$

The constant factors in the above inequalities are the best possible.

\section{Conclusions}

In this paper, according to the way of [21, 22], by applying the weight functions, the idea of introduced parameters, and the Euler-Maclaurin summation formula, a new extended half-discrete Hilbert's inequality with the homogeneous kernel and the beta, gamma function is given in Theorem 1. The preliminaries are obtained in Theorem 2. The equivalent statements of the best possible constant factor related to some parameters are proved in Theorem 3. As applications, a corollary about the case of the non-homogeneous kernel and some particular cases are considered in Corollary 1 and Remark 4. The lemmas and theorems provide an extensive account of this type of inequalities.

\section{Acknowledgements}

The authors thank the referee for his useful suggestions to reform the paper.

\section{Funding}

This work is supported by the National Natural Science Foundation (Nos. 11961021, 11561019) and Hechi University Research Fund for Advanced Talents (No. 2019GC005). We are grateful for this help.

\section{Availability of data and materials}

The data and material in this paper are effective.

\section{Competing interests}

The authors declare that they have no competing interests.

\section{Authors' contributions}

BY carried out the mathematical studies, participated in the sequence alignment, and drafted the manuscript. XH and RL participated in the design of the study and performed the numerical analysis. All authors read and approved the final manuscript.

\section{Author details}

'School of Mathematics and Statistics, Hechi University, Yizhou, P.R. China. ${ }^{2}$ Department of Mathematics, Guangdong University of Education, Guangzhou, P.R. China.

\section{Publisher's Note}

Springer Nature remains neutral with regard to jurisdictional claims in published maps and institutional affiliations.

Received: 28 September 2019 Accepted: 21 January 2020 Published online: 30 January 2020

\section{References}

1. Hardy, G.H., Littlewood, J.E., Polya, G.: Inequalities. Cambridge University Press, Cambridge (1934)

2. Yang, B.C.: The Norm of Operator and Hilbert-Type Inequalities. Science Press, Beijing (2009)

3. Yang, B.C.: Hilbert-Type Integral Inequalities. Bentham Science Publishers Ltd., Sharjah (2009)

4. Yang, B.C.: On the norm of an integral operator and applications. J. Math. Anal. Appl. 321, 182-192 (2006)

5. Xu, J.S.: Hardy-Hilbert's inequalities with two parameters. Adv. Math. 36(2), 63-76 (2007)

6. Yang, B.C.: On the norm of a Hilbert's type linear operator and applications. J. Math. Anal. Appl. 325, 529-541 (2007)

7. Xie, Z.T., Zeng, Z., Sun, Y.F.: A new Hilbert-type inequality with the homogeneous kernel of degree -2. Adv. Appl. Math. Sci. 12(7), 391-401 (2013)

8. Zhen, Z., Raja Rama Gandhi, K., Xie, Z.T.: A new Hilbert-type inequality with the homogeneous kernel of degree -2 and with the integral. Bull. Math. Sci. Appl. 3(1), 11-20 (2014)

9. Xin, D.M.: A Hilbert-type integral inequality with the homogeneous kernel of zero degree. Math. Theory Appl. 30(2), 70-74 (2010)

10. Azar, L.E.: The connection between Hilbert and Hardy inequalities. J. Inequal. Appl. 2013, 452 (2013)

11. Batbold, T., Sawano, Y.: Sharp bounds for $m$-linear Hilbert-type operators on the weighted Morrey spaces. Math. Inequal. Appl. 20, 263-283 (2017) 
12. Adiyasuren, V., Batbold, T., Krnic, M.: Multiple Hilbert-type inequalities involving some differential operators. Banach J. Math. Anal. 10, 320-337 (2016)

13. Adiyasuren, V., Batbold, T., Krnić, M.: Hilbert-type inequalities involving differential operators, the best constants and applications. Math. Inequal. Appl. 18, 111-124 (2015)

14. Rassias, M.T., Yang, B.C.: On half-discrete Hilbert's inequality. Appl. Math. Comput. 220, 75-93 (2013)

15. Yang, B.C., Krnić, M.: A half-discrete Hilbert-type inequality with a general homogeneous kernel of degree 0. J. Math. Inequal. 6(3), 401-417 (2012)

16. Rassias, M.T., Yang, B.C.: A multidimensional half-discrete Hilbert-type inequality and the Riemann zeta function. Appl. Math. Comput. 225, 263-277 (2013)

17. Rassias, M.T., Yang, B.C.: On a multidimensional half-discrete Hilbert-type inequality related to the hyperbolic cotangent function. Appl. Math. Comput. 242, 800-813 (2013)

18. Huang, Z.X., Yang, B.C.: On a half-discrete Hilbert-type inequality similar to Mulholland's inequality. J. Inequal. Appl. 2013, $290(2013)$

19. Yang, B.C., Lebnath, L.: Half-Discrete Hilbert-Type Inequalities. World Scientific, Singapore (2014)

20. Krnić, M., Pečarić, J.: Extension of Hilbert's inequality. J. Math. Anal. Appl. 324(1), 150-160 (2006)

21. Adiyasuren, V., Batbold, T., Azar, L.E.: A new discrete Hilbert-type inequality involving partial sums. J. Inequal. Appl. 2019, $127(2019)$

22. Hong, Y., Wen, Y.: A necessary and sufficient condition of that Hilbert type series inequality with homogeneous kernel has the best constant factor. Ann. Math. 37A(3), 329-336 (2016)

23. Hong, Y:: On the structure character of Hilbert's type integral inequality with homogeneous kernel and applications. J. Jilin Univ. Sci. Ed. 55(2), 189-194 (2017)

24. Hong, Y., Huang, Q.L., Yang, B.C., Liao, J.L.: The necessary and sufficient conditions for the existence of a kind of Hilbert-type multiple integral inequality with the non-homogeneous kernel and its applications. J. Inequal. Appl. 2017, $316(2017)$

25. Xin, D.M., Yang, B.C., Wang, A.Z.: Equivalent property of a Hilbert-type integral inequality related to the beta function in the whole plane. J. Funct. Spaces 2018, Article ID 2691816 (2018)

26. Hong, Y., He, B., Yang, B.C.: Necessary and sufficient conditions for the validity of Hilbert type integral inequalities with a class of quasi-homogeneous kernels and its application in operator theory. J. Math. Inequal. 12(3), 777-788 (2018)

27. Kuang, J.C.: Applied Inequalities. Shangdong Science and Technology Press, Jinan (2004)

28. Kuang, J.C.: Real and Functional Analysis (Continuation), vol. 2. Higher Education Press, Beijing (2015)

\section{Submit your manuscript to a SpringerOpen ${ }^{\circ}$ journal and benefit from:}

- Convenient online submission

- Rigorous peer review

- Open access: articles freely available online

- High visibility within the field

- Retaining the copyright to your article

Submit your next manuscript at $\boldsymbol{~ s p r i n g e r o p e n . c o m ~}$ 